\title{
Unilateral Moyamoya Phenomenon with a String-of-beads Appearance in an Elderly Patient with the c.14576G $>$ A Heterozygous Variant of RNF213
}

\author{
Junya Aoki ${ }^{1}$, Kensaku Shibazaki ${ }^{1}$, Masaki Ito ${ }^{2}$, Naoki Saji ${ }^{1}$, Junichi Uemura ${ }^{1}$, \\ Kiyohiro Houkin ${ }^{2}$ and Kazumi Kimura ${ }^{1}$
}

\begin{abstract}
We herein report a case of ischemic stroke in a 69-year-old man with unilateral moyamoya vessels originating from the proximal portion of the left middle cerebral artery. In addition, digital-subtraction angiography demonstrated a string-of-beads-like appearance in the cavernous portion of the left internal carotid artery. A genetic analysis revealed a heterozygous c. $14576 \mathrm{G}>\mathrm{A}$ variant in ring finger protein 213 . The patient's younger brother had a history of hemorrhagic stroke and had been diagnosed with moyamoya disease. We finally considered that the unilateral moyamoya vessel and string-of-beads appearance observed in the current case were not simply caused by atherosclerosis, but rather represented symptoms within the moyamoya spectrum.
\end{abstract}

Key words: moyamoya vessel, RNF213, string-of-beads appearance

(Intern Med 54: 971-974, 2015)

(DOI: 10.2169/internalmedicine.54.3534)

\section{Introduction}

Moyamoya disease is characterized by the presence of bilateral stenosis or occlusion of the terminal portion of the intracranial internal carotid artery (ICA) or proximal portion of the anterior cerebral artery (ACA) and/or middle cerebral artery (MCA). Abnormal collateral vessels exhibiting a "puff of smoke" appearance (moyamoya vessels) are observed in the vicinity of such arterial lesions (1). The ring finger protein 213 (RNF213) gene was recently identified to indicate susceptibility to moyamoya disease (2). We herein report the case of an elderly man with ischemic stroke and a heterozygous variant of RNF213 who exhibited a unilateral moyamoya vessel and string-of-beads-like appearance in the ipsilateral ICA.

\section{Case Report}

A 69-year-old righted-handed man was admitted to our hospital with a disturbance of consciousness and right hemiparesis. Before being brought to our hospital, he had been rescued from a fire in his apartment by firefighters. The patient had a history of hypertension, drinking and smoking. He was $167 \mathrm{~cm}$ tall and weighed $52.4 \mathrm{~kg}$. Upon admission, his body temperature was $37.6^{\circ} \mathrm{C}$, his blood pressure was $170 / 106 \mathrm{mmHg}$ and his heart rate was 106 beats/min with a regular sinus rhythm. No cardiac murmurs or carotid bruits were audible, and the skin appeared normal. A neurological examination revealed a disturbance of consciousness (Glasgow Coma Scale, 12), motor aphasia, right horizontal gaze palsy, right central facial palsy, dysarthria and right hemiparesis. An extensor plantar response was present on the right side, and the National Institutes of Health Stroke Scale score was 16 . The laboratory data showed an elevated blood cell count, with a white blood cell count of $11,440 / \mu \mathrm{L}$, a hemoglobin level of $17.8 \mathrm{~g} / \mathrm{dL}$ and platelet count of $34.1 \times 10^{4} /$ $\mu \mathrm{L}$. The serum blood glucose level was $137 \mathrm{mg} / \mathrm{dL}$, the hemoglobin A1c level was 5.7\% and the low-density lipoprotein cholesterol level was $244 \mathrm{mg} / \mathrm{dL}$. In addition, the inter-

${ }^{1}$ Department of Stroke Medicine, Kawasaki Medical School, Japan and ${ }^{2}$ Department of Neurosurgery, Hokkaido University Graduate School of Medicine, Japan

Received for publication June 25, 2014; Accepted for publication September 10, 2014

Correspondence to Dr. Junya Aoki, aokijy@gmail.com 

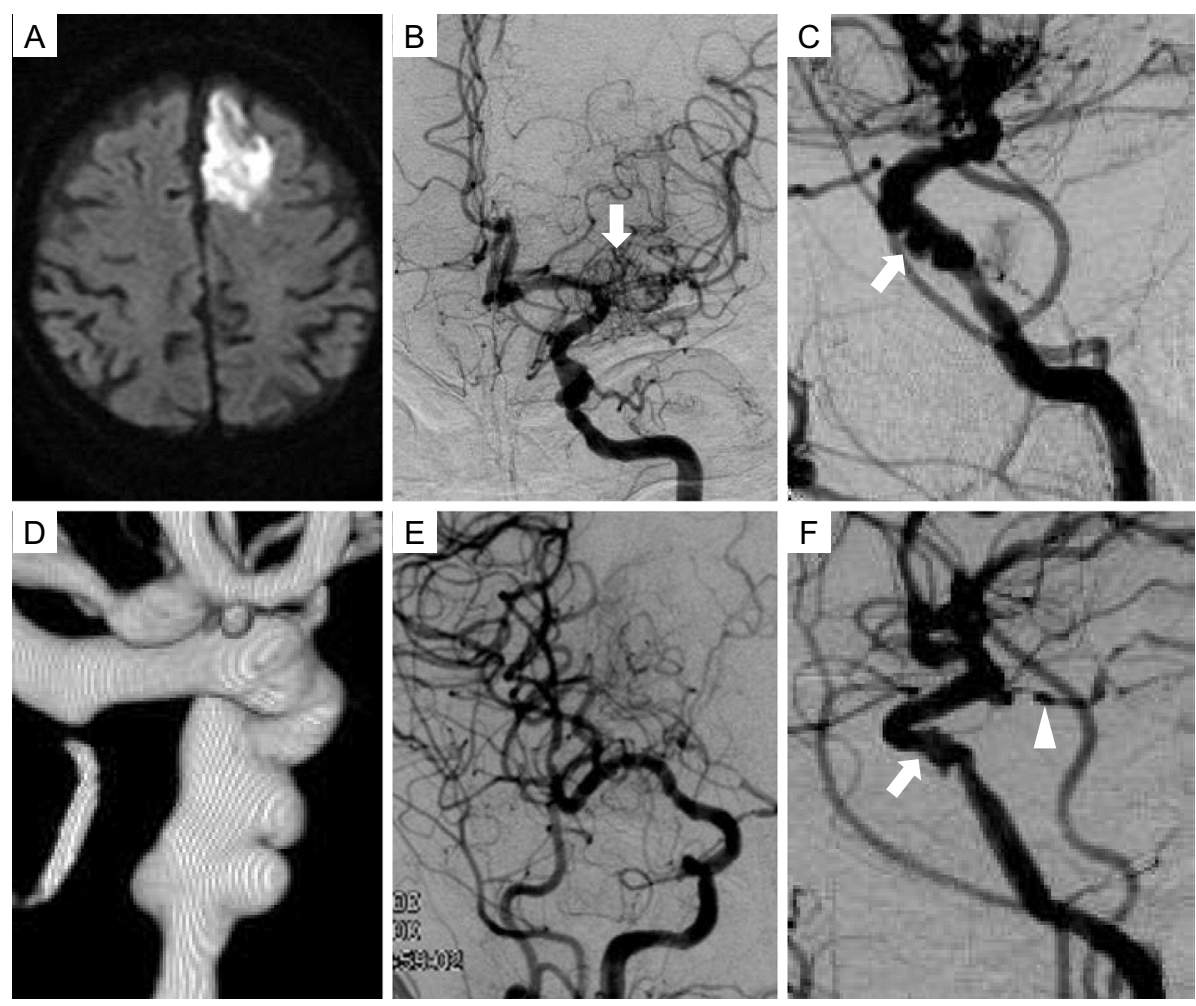

Figure 1. A: Diffusion-weighted imaging shows a hyperintense lesion on the left side of the frontal lobe. B: Digital-subtraction angiography (DSA) demonstrates moyamoya vessels (arrow) originating from the proximal portion of the left middle cerebral artery. C: Repeated dilated lesions (arrow) are observed in the cavernous segment of the left internal carotid artery. D: Three-dimensional DSA of the dilated lesions. E: No occlusion is present in the contralateral middle cerebral artery. F: Dilated lesions are also seen in the right internal carotid artery (arrow) and posterior cerebral artery (arrowhead).

national normalized ratio was 1.12 , the activated partial thromboplastin time was $24.4 \mathrm{~s}$ and the D-dimer level was $1.9 \mu \mathrm{g} / \mathrm{mL}$. The patient's thyroid function was normal, with a free T4 level of $1.13 \mathrm{ng} / \mathrm{dL}$, free T3 level of $2.51 \mathrm{pg} / \mathrm{mL}$ and thyroid-stimulating hormone level of $0.85 \mu \mathrm{U} / \mathrm{mL}$. The protein $\mathrm{C}$ and $\mathrm{S}$ levels were also within the normal limits, and tests for antithrombin, anticardiolipin antibodies, antinuclear antibodies, anti-DNA antibodies, lupus anticoagulant and anti- $\beta 2$ GPI antibodies were negative. Carotid ultrasonography showed no significant stenosis. The patient was subsequently examined using a 1.5-T magnetic resonance unit (Signa EchoSpeed Horizon, GE Medical Systems, Milwaukee, USA), and diffusion-weighted imaging (DWI) showed hyperintense lesions in the right cerebellum and on the left side of the frontal lobe. Furthermore, occlusion of the left ACA and MCA was seen on magnetic resonance angiography (MRA), and digital-subtraction angiography (DSA) demonstrated collateral vessels, showing a moyamoya vessel originating from the left proximal MCA (Fig. 1). Moreover, DSA revealed dilated lesions in the cavernous portion of the left ICA, representing the so-called "string-of-beads" phenomenon. Although mild, a similar appearance was apparent in the contralateral ICA. Comparable changes were also present in the right posterior cerebral artery; however, no occlusion or similar findings were noted in the contralateral MCA. Transthoracic echocardiography showed stenosis of the middle left ventricle, and computed tomography angiography identified serial calcification of the abdominal aorta. Based on the patient's advanced age and history of smoking and drinking and the findings of unilateral occlusion and arteriosclerotic changes in the heart and aorta, we initially diagnosed him with ischemic stroke due to ACA occlusion. The etiology of both the MCA and ACA occlusion was considered to be atherosclerosis, although the dilated lesions were atypical.

The family history elicited from his nephew revealed that the patient's 61-year-old brother had experienced hemorrhagic stroke at 47 years of age; this brother had been injured in the same fire and transferred to another hospital. We subsequently examined him using MRA and identified bilateral ICA occlusion. Interestingly, a dilated appearance was observed in the petrous and cervical portions of the left ICA. In addition, he had been diagnosed with moyamoya disease after DSA performed at 47 years of age revealed moyamoya vessels in the bilateral basal ganglia (Fig. 2).

Based on these findings, we suspected that our elderly patient had developed ischemic-onset quasi-moyamoya disease. After obtaining written informed consent from his legal representatives, a genetic analysis was performed (M.I. and K. H. at Hokkaido University). DNA specimens purified from 


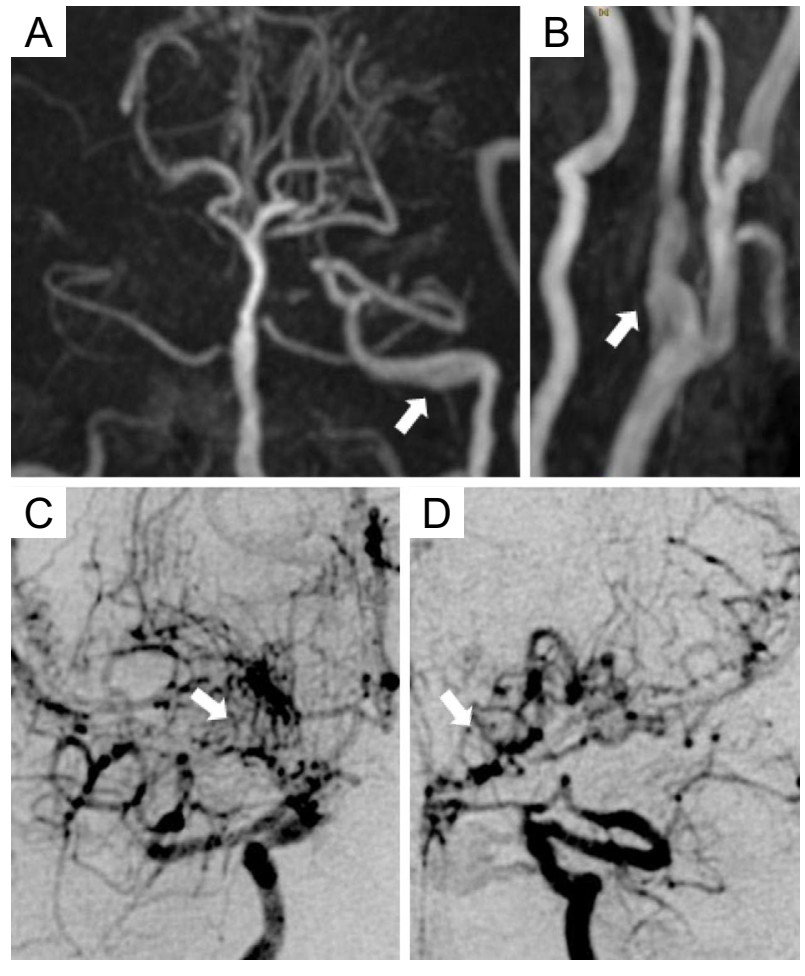

Figure 2. A, B: Magnetic resonance angiography of the younger brother shows bilateral ICA occlusion as well as a dilated lesion (arrows) in the cervical segment of the left internal carotid artery. C, D: Cerebral angiogram of the younger brother at 47 years of age shows moyamoya vessels (arrows) in the basal ganglia bilaterally.

the patient's blood samples revealed the c.14576G $>$ A heterozygous variant in RNF213 (Fig. 3). As a result, we finally considered that the unilateral moyamoya vessel and string-of-beads appearance noted in this case were not simply caused by atherosclerosis, but rather represented symptoms of the moyamoya spectrum.

\section{Discussion}

We herein reported a case of ischemic stroke associated with heterozygous c.14576G $>\mathrm{A}$ polymorphism in RNF213 in which the patient displayed a unilateral moyamoya vessel and a dilated lesion showing a string-of-beads appearance in the ICA.

The patient in this case had several risk factors for atherosclerosis, including an advanced age and history of hypertension, smoking and drinking. In addition, systematic atherosclerotic changes were seen in the heart and abdominal aorta. Atherosclerosis is a well-known differential diagnosis of moyamoya disease (1). Atherosclerotic risk factors have previously been reported to be more frequent in patients with unilateral moyamoya vessels than in those with moyamoya disease (3). Although some cases of unilateral moyamoya vessels progress to the bilateral form, this phenomenon is primarily observed within 1-2 years in childhood. The possibility of progression in adolescence and be-
A
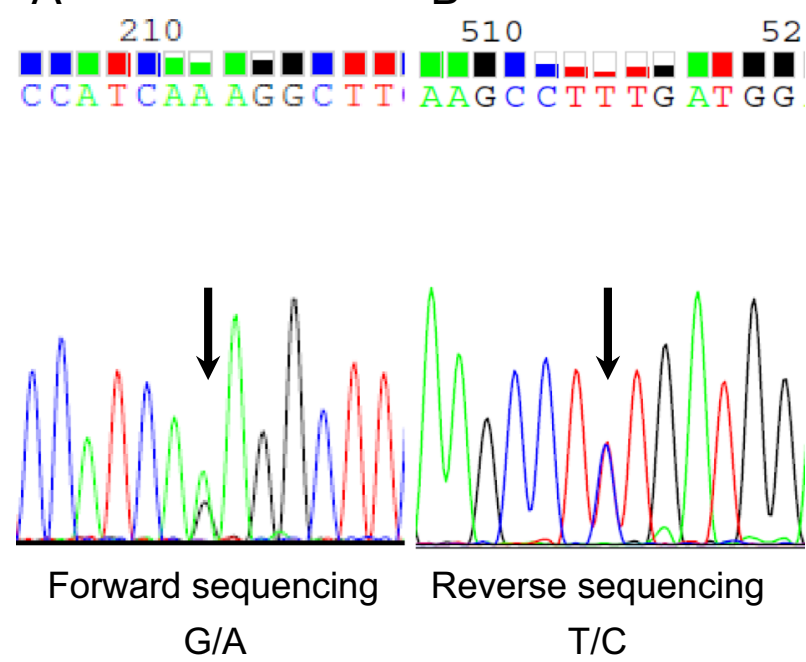

Figure 3. Sequencing chromatograms (A: forward sequence, $B$ : reverse sequence) showing the $14576 G>A$ heterozygous variant in RNF213 (arrows).

yond appears to be infrequent $(4,5)$. The level of basic fibroblast growth factor in the cerebrospinal fluid is reported to be related to progression (6). It thus is reasonable to consider that the presence of unilateral moyamoya vessels in elderly individuals is attributable to atherosclerosis and that this phenomenon is distinct from moyamoya disease.

The present elderly patient with a unilateral moyamoya vessel had a family history of moyamoya disease. In addition, a genetic analysis revealed the presence of the risk allele in RNF213. Liu et al. identified RNF213 as a susceptibility gene for moyamoya disease, showing that a knockdown zebrafish model results in the formation of vessels with an irregular diameter that exhibit aberrant sprouting (2). In a recent study by Miyawaki et al., over $80 \%$ of moyamoya disease patients were found to have the variant RNF213, (7) although no significant associations with extracranial carotid atherosclerosis, cerebral aneurysm development or intracerebral hemorrhage were identified. Intriguingly, the authors also reported that $22 \%$ of patients with unilateral ICA occlusion or stenosis display the variant RNF 213. Patients diagnosed with the uni- or bilateral form of the condition may not necessarily present with the pathogenesis of moyamoya disease. Current guidelines do not confirm the present case to be moyamoya disease due to the presence of unilateral occlusion. However, we believe that genetic analyses of RNF213 provide a key to understanding the etiology of the disease, even in elderly patients with unilateral moyamoya vessels and siblings who present with definitive moyamoya disease.

In the current case, a dilated lesion with a string-of-beads appearance was observed in the cavernous segment of the ICA. Although the severity of the condition differed, his younger brother also exhibited dilated lesions in the ICA. These findings are not in line with the pathological consensus that moyamoya disease involves the presence of occlu- 
sion or stenosis in the terminal portion of the ICA (8). However, several case reports have described dilated lesions. For example, Khan et al. reported the cases of two unrelated children with moyamoya disease who showed bilateral dolichoectatic ICA lesions. Both patients also presented with pupillary dysfunction and cardiac malformations (9). Cases of moyamoya vessels originating from dolichoectatic arteries have also been reported $(10,11)$. Although genetic abnormalities, infection, environmental factors, cytokines and autoimmune mechanisms are all presumed to be associated with the pathogenesis of moyamoya disease, the etiology of this disorder has yet to be fully elucidated. We presume that the similarity in dilated appearance between our patient and previously reported cases indicates the presence of an unknown factor that organizes dilated lesions in patients with moyamoya disease.

In conclusion, the present report suggests that some cases of unilateral moyamoya vessels fall within the spectrum of moyamoya disease, even if the patient exhibits several atherosclerotic risk factors and dilated lesions.

The authors state that they have no Conflict of Interest (COI).

\section{Acknowledgement}

We are grateful to Yumiko Shinohe (Neurosurgery, Hokkaido University) and Yuuka Hama (Neurology, Hokkaido University) for conducting the analysis of the patient's genetic mutation.

\section{References}

1. Guidelines for diagnosis and treatment of moyamoya disease (spontaneous occlusion of the circle of Willis). Neurol Med Chir (Tokyo) 52: 245-266, 2012.

2. Liu W, Morito D, Takashima S, et al. Identification of RNF213 as a susceptibility gene for moyamoya disease and its possible role in vascular development. PLoS One 6: e22542, 2011.

3. Ogata T, Yasaka M, Inoue $T$, et al. The clinical features of adult unilateral moyamoya disease: does it have the same clinical characteristics as typical moyamoya disease? Cerebrovasc Dis 26: 244249, 2008.

4. Kawano T, Fukui M, Hashimoto N, Yonekawa Y. Follow-up study of patients with "unilateral" moyamoya disease. Neurol Med Chir (Tokyo) 34: 744-747, 1994.

5. Yeon JY, Shin HJ, Kong DS, et al. The prediction of contralateral progression in children and adolescents with unilateral moyamoya disease. Stroke 42: 2973-2976, 2011.

6. Houkin K, Abe H, Yoshimoto T, Takahashi A. Is "unilateral" moyamoya disease different from moyamoya disease? J Neurosurg 85: 772-776, 1996.

7. Miyawaki S, Imai H, Takayanagi S, Mukasa A, Nakatomi H, Saito $\mathrm{N}$. Identification of a genetic variant common to moyamoya disease and intracranial major artery stenosis/occlusion. Stroke 43: 3371-3374, 2012.

8. Suzuki J, Takaku A. Cerebrovascular "moyamoya" disease. Disease showing abnormal net-like vessels in base of brain. Arch Neurol 20: 288-299, 1969.

9. Khan N, Schinzel A, Shuknecht B, Baumann F, Ostergaard JR, Yonekawa Y. Moyamoya angiopathy with dolichoectatic internal carotid arteries, patent ductus arteriosus and pupillary dysfunction: a new genetic syndrome? Eur Neurol 51: 72-77, 2004.

10. Yamada K, Hayakawa T, Ushio Y, Mitomo M. Cerebral arterial dolichoectasia associated with moyamoya vessels. Surg Neurol 23: 19-24, 1985.

11. Hanakita J, Miyake H, Nagayasu S, Nishi S, Suzuki T. Surgically treated cerebral arterial ectasia with so-called moyamoya vessels. Neurosurgery 19: 271-273, 1986.

(C) 2015 The Japanese Society of Internal Medicine http://www.naika.or.jp/imonline/index.html 